\title{
A Magnetically Controlled Soft Microrobot Steering a Guidewire in a Three-Dimensional Phantom Vascular Network
}

\author{
Sungwoong Jeon, ${ }^{1,2, *}$ Ali Kafash Hoshiar, ${ }^{1,2, *}$ Kangho Kim, ${ }^{1,2, *}$ Seungmin Lee,,2 \\ Eunhee Kim, ${ }^{1,2}$ Sunkey Lee,, Jin-young Kim, ${ }^{1,2}$ Bradley J. Nelson, ${ }^{2,3}$ Hyo-Jeong Cha, \\ Byung-Ju $\mathrm{Yi}^{4}$, and Hongsoo Choi ${ }^{1,2}$
}

\begin{abstract}
Magnetically actuated soft robots may improve the treatment of disseminated intravascular coagulation. Significant progress has been made in the development of soft robotic systems that steer catheters. A more challenging task, however, is the development of systems that steer sub-millimeter-diameter guidewires during intravascular treatments; a novel microrobotic approach is required for steering. In this article, we develop a novel, magnetically actuated, soft microrobotic system, increasing the steerability of a conventional guidewire. The soft microrobot is attached to the tip of the guidewire, and it is magnetically steered by changing the direction and intensity of an external magnetic field. The microrobot is fabricated via replica molding and features a soft body made of polydimethylsiloxane, two permanent magnets, and a microspring. We developed a mathematical model mapping deformation of the soft microrobot using a feed-forward approach toward steering. Then, we used the model to steer a guidewire. The angulation of the microrobot can be controlled from $21.1^{\circ}$ to $132.7^{\circ}$ by using a magnetic field of an intensity of $15 \mathrm{mT}$. Steerability was confirmed by two-dimensional in vitro tracking. Finally, a guidewire with the soft microrobot was tested by using a three-dimensional (3D) phantom of the coronary artery to verify steerability in 3D space.
\end{abstract}

Keywords: soft microrobot, guidewire, percutaneous coronary intervention (PCI), steerability, intravascular treatments, magnetic steering

\section{Introduction}

$\mathbf{O}$ VER THE PAST FEW YEARS, untethered microrobots have found diverse applications in biopsy, ${ }^{1,2}$ treatment of hyperthermia, ${ }^{3}$ cell culture, ${ }^{4,5}$ targeted drug delivery, ${ }^{6-8}$ tissue scaffolding, ${ }^{9}$ sensing, ${ }^{10}$ tissue marking, ${ }^{11}$ and microsurgery. ${ }^{12-14}$ More recently, soft micromanipulators have been used for targeted drug delivery. ${ }^{15}$ The soft deformable structures of such microrobots render them well suited to perform biomedical operations within vascular networks.

Cardiovascular diseases were responsible for one of every three deaths worldwide in 2013, and chronic total occlusions are found in about one-third of patients with coronary artery disease. ${ }^{16,17}$ Percutaneous coronary intervention (PCI) (a minimally invasive procedure) can be used to unblock coronary arteries. ${ }^{18-20}$ PCI features the use of guidewires and catheters. PCI is performed by inserting a catheter into the femoral or radial artery and steering that catheter into the heart. The process is monitored by using an X-ray imaging system (angiography). The catheter is positioned inside the coronary artery, and the $\mathrm{X}$ ray image is used to map the arteries and locate the blockage or narrowing. Then, a guidewire is inserted into the artery and steered to the target position. If necessary, a balloon catheter can be delivered to the target position and the artery is inflated.

\footnotetext{
${ }^{1}$ Department of Robotics Engineering, Daegu Gyeongbuk Institute of Science and Technology (DGIST), Daegu, South Korea.

${ }^{2}$ DGIST-ETH Microrobotics Research Center (DEMRC), DGIST, Daegu, South Korea.

${ }^{3}$ Institute of Robotics and Intelligent Systems, ETH Zurich, Zurich, Switzerland.

${ }^{4}$ Department of Electronic Systems Engineering, Hanyang University, Ansan, Korea.

*These authors contributed equally to this work.
}

(C) Sungwoong Jeon et al. 2019; Published by Mary Ann Liebert, Inc. This Open Access article is distributed under the terms of the Creative Commons License (http://creativecommons.org/licenses/by/4.0), which permits unrestricted use, distribution, and reproduction in any medium, provided the original work is properly cited. 
During this procedure, conventional guidewires are manually controlled by back-and-forth pushing and rotating. This prolongs the operation time and increases the radiation exposure of both patients and doctors. Further, the procedural success rate is highly dependent on the skill and experience of the physician. Thus, a number of research groups have attempted to develop steerable guidewire systems, as discussed later.

The use of elastic tubes has been studied in this context. ${ }^{21}$ However, contact with vessel walls and the associated friction, which compromise precision, have imposed limits on their application. Shape memory alloy actuators $<2 \mathrm{~mm}$ in diameter have been used to steer catheters. ${ }^{22}$ However, the maximal bending angle was $<90^{\circ}$, and the need for submillimeter design remained unanswered. More recently, soft actuators made of various materials have been developed to steer catheters. ${ }^{23}$ Eight deformations were programmed by using a microcontroller, and a 10-g load was used to evaluate performance. Further, a scaled version of a three-dimensional (3D)-printed carotid artery was utilized for steering. Despite successful proof-of-concept, temperature and size limitations remain. Magnetically controlled catheters have attracted significant attention over the past few years because of their precise and responsive steerability.

Magnetic resonance imaging (MRI)-based actuation has been used to remotely steer and monitor catheters, ${ }^{24}$ and the relationship between magnetic torque and the mechanical restoration of torque has been modeled. ${ }^{25}$ However, the model employs linear terms for simplicity and, thus, fails to predict nonlinear deformations. Further, the MRI-based systems are not tailored to endovascular interventions. Magnetic actuation systems for steering catheters have been proposed, ${ }^{26,27}$ as have position control schemes. ${ }^{28,29}$ However, guidewire steering is needed for many intravascular applications, such as PCI.

In a previous study, a magnet was attached to the tip of a guidewire and two permanent magnets positioned at either a

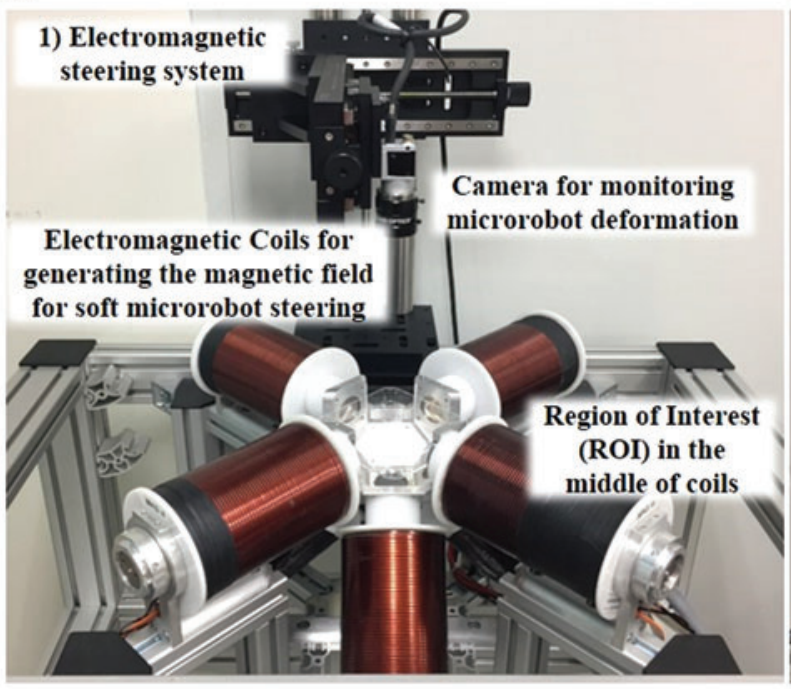

C

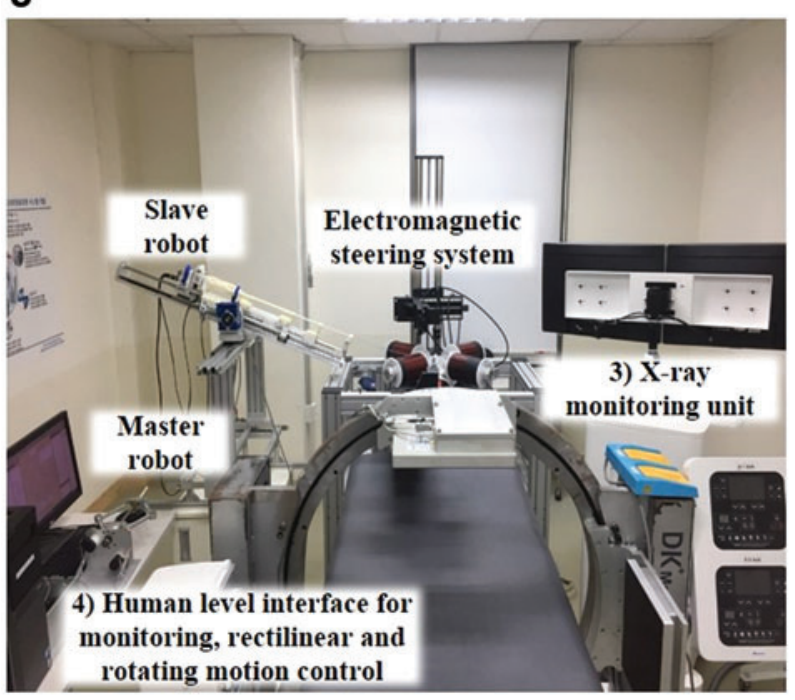

b

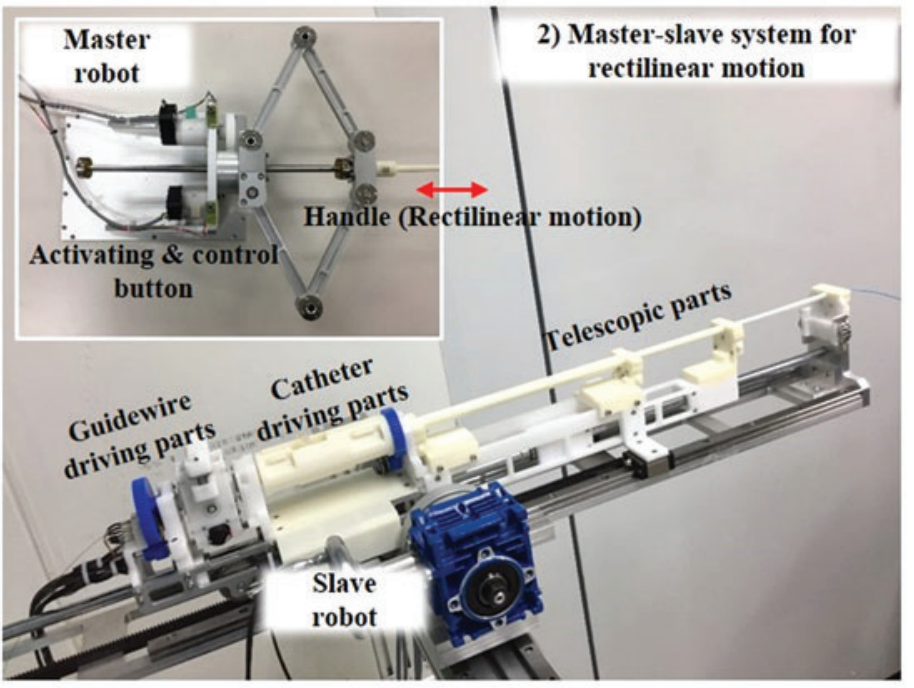

d

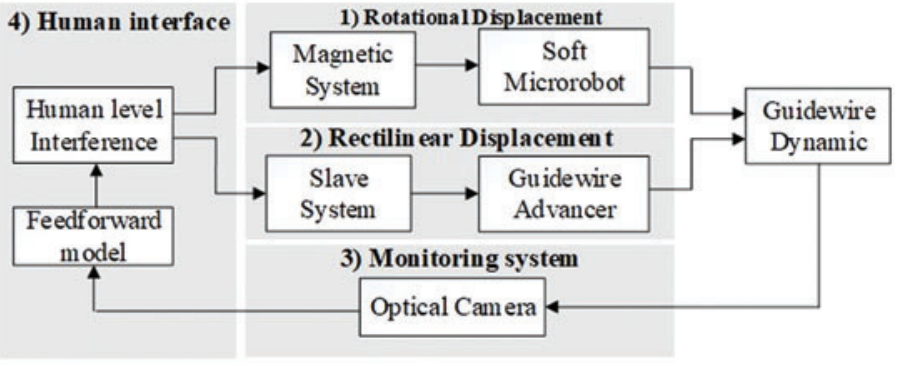

FIG. 1. Overview of the magnetically actuated microrobotic system. (a) The OctoMag actuation system for microrobot steering; (b) the master/slave robotic system controlling rectilinear motion; (c) the X-ray monitoring system (not used in the present work) and the user interface; and (d) the schematic and the connections for guidewire steering. Color images are available online. 
side of a fluoroscopy table were used to steer the guidewire. ${ }^{30}$ Although the guidewire became aligned with the direction of the field, the use of permanent magnets for steering limits the ability to control the magnetic field. MRI-based guidewire steering has also been studied, but image reconstruction is time-consuming and the need for sequential imaging/steering compromises the utility of the approach. ${ }^{31,32}$ Therefore, we developed a soft submillimeter-diameter microrobot mounted at the end of a conventional guidewire. The microrobot is steered by using a dedicated actuation system consisting of eight electromagnets.

We used polydimethylsiloxane (PDMS, Sylgard 184; Dow Corning Corp., Midland, MI), which has a low elastic modulus and a high Poisson ratio, to fabricate the soft microrobot that incorporates two permanent magnets, allowing steering by an external magnetic field within a phantom vascular network. We developed a mathematical model of the relationship between the magnetic force and microrobot deformation. As this was nonlinear, the force-deformation relationship was modeled via finite element analysis (FEA). Deformation angles of up to $132.7^{\circ}$ were experimentally achieved. Two-dimensional (2D) and 3D tracking experiments showed that steering and tracking performance was reliable.

\section{Materials and Methods}

\section{The soft microrobot}

The steering system has four major components (Fig. 1). A magnetic field steers the soft microrobot. Figure 1a shows the magnetic actuation system (OctoMag; Aeon Scientific $\mathrm{GmbH}$, Schlieren, Switzerland), which consists of eight electromagnets generating a 3D magnetic field of a maximum intensity of $40 \mathrm{mT}$ (for details see Kummer et al. ${ }^{12}$ ). Guidewire rectilinear motion is created by using a master/ slave system (Fig. 1b). The master is activated by the operator, and the slave generates rectilinear motion. The system can be used to remotely control commercial guidewires and catheters intravascularly (for details Cha et al. ${ }^{33}$ ). Once the microrobot points in the desired direction, the slave moves forward and advances the guidewire and the microrobot.

The complete system, shown in Figure 1c, features an Xray system enabling real-time monitoring similar to that of conventional PCI. In this study, however, optical monitoring was employed for convenience; X-ray monitoring will be studied in future works.

Figure 1d shows an overview of the system and its connections. Rotational and rectilinear displacements are triggered via magnetic actuation and the master/slave system, respectively. Feedback is provided by a camera. We developed a model of the rectilinear and angular displacements, and a user interface to control both processes.

\section{The magnetic steering system}

The magnetic steering system consists of the OctoMag system and the novel soft microrobot (Fig. 2). Two permanent magnets were placed at equal distance within the PDMS matrix and used to steer the guidewire. The OctoMag actuation system $^{12}$ can generate a $3 \mathrm{D}$ magnetic field of constant magnitude that varies in terms of direction. As the field direction changes, the microrobot experiences a magnetic torque, forcing realignment in the direction of the field. Thus,

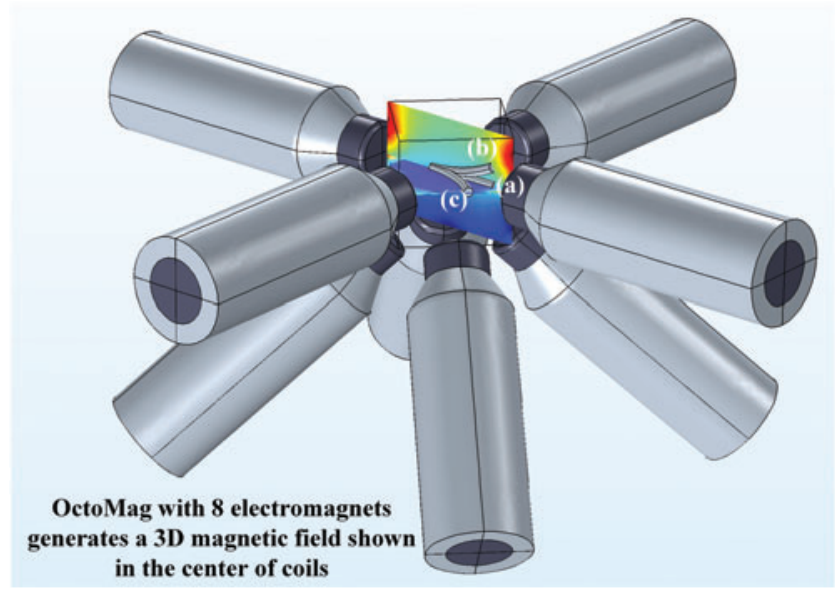

FIG. 2. A schematic of the OctoMag system ${ }^{12}$ and the soft microrobot. The microrobot is steered by changes in the direction of the magnetic field. The microrobot dimensions are exaggerated to afford better visualization; the microrobot is illustrated in three different positions: (a) the initial position; (b) after deformation in the $z$ direction; and (c) after deformation in the $x$ direction. Color images are available online.

the microrobot is magnetically steered in the direction of interest (Fig. 2).

\section{Model of Magnetic Actuation}

\section{Mathematical model}

The magnetic field in current-free space is described by Maxwell's equations as:

$$
\begin{aligned}
& \nabla \cdot \vec{B}=0 \\
& \nabla \times \vec{B}=0
\end{aligned}
$$

where $\vec{B}$ is the $3 \times 1$ magnetic field vector. Considering the boundary conditions, these equations describe the magnetic field. The force and torque on a magnetic object in this magnetic field are given by the following equations:

$$
\begin{gathered}
\vec{F}=(\vec{m} \cdot \nabla) \vec{B} \\
\vec{\tau}=\vec{m} \times \vec{B}
\end{gathered}
$$

where $\vec{F}$ and $\vec{\tau}$ are $3 \times 1$ force and torque vectors, respectively, and $\vec{m}$ is the $3 \times 1$ dipole moment of the magnetic object. Maxwell's equations impose the following two constraints: (1) Equation (1) indicates that the gradient matrix has a zero trace, and (2) Equation (2) shows that the gradient matrix is symmetrical. Therefore, Equation (3) can be written as:

$$
\vec{F}=\left[\begin{array}{l}
f_{x} \\
f_{y} \\
f_{z}
\end{array}\right]=\left[\begin{array}{ccc}
\frac{\partial \mathrm{B}_{x}}{\partial x} & \frac{\partial \mathrm{B}_{x}}{\partial y} & \frac{\partial \mathrm{B}_{x}}{\partial z} \\
\frac{\partial \mathrm{B}_{x}}{\partial y} & \frac{\partial \mathrm{B}_{y}}{\partial y} & \frac{\partial \mathbf{B}_{y}}{\partial x} \\
\frac{\partial \mathrm{B}_{x}}{\partial z} & \frac{\partial \mathbf{B}_{y}}{\partial z} & -\left(\frac{\partial \mathrm{B}_{y}}{\partial z}+\frac{\partial \mathrm{B}_{x}}{\partial x}\right)
\end{array}\right]\left[\begin{array}{c}
m_{x} \\
m_{y} \\
m_{z}
\end{array}\right]
$$


The magnetic field gradient represented by Equation (5) has five components, which can be represented as a $5 \times 1$ matrix $\left(B_{g}\right)$. Consequently, Equation (5) can be rearranged as:

$$
\begin{aligned}
\vec{F}=\left[\begin{array}{l}
f_{x} \\
f_{y} \\
f_{z}
\end{array}\right] & =\left[\begin{array}{ccccc}
m_{x} & m_{y} & m_{z} & 0 & 0 \\
0 & m_{x} & 0 & m_{y} & m_{z} \\
-m_{z} & 0 & m_{x} & -m_{z} & m_{y}
\end{array}\right]\left[\begin{array}{c}
\frac{\partial B_{x}}{d x} \\
\frac{\partial B_{x}}{d y} \\
\frac{\partial B_{x}}{d z} \\
\frac{\partial B_{y}}{d y} \\
\frac{\partial B_{y}}{d z}
\end{array}\right] \\
& =M_{F} B_{g}
\end{aligned}
$$

where $M_{F}$ packs the dipole terms contributing to the magnetic force. Considering the cross-product in Equation (4), the magnetic torque vector can be represented as:

$$
\begin{aligned}
\vec{\tau}=\left[\begin{array}{c}
\tau_{x} \\
\tau_{y} \\
\tau_{z}
\end{array}\right] & =\left[\begin{array}{ccc}
0 & -m & m_{y} \\
m_{z} & 0 & -m_{x} \\
-m_{y} & m_{x} & 0
\end{array}\right]\left[\begin{array}{l}
B_{x} \\
B_{y} \\
B_{z}
\end{array}\right] \\
& =M_{\tau} B
\end{aligned}
$$

$$
\left[\begin{array}{l}
\tau \\
F
\end{array}\right]_{6 \times 1}=\left[\begin{array}{cc}
M_{\tau} & 0 \\
0 & M_{F}
\end{array}\right]_{6 \times 8}\left[\begin{array}{l}
B \\
B_{g}
\end{array}\right]_{8 \times 1}
$$

The system outputs (torque and force) depend on this $6 \times 1$ matrix. When investigating the soft microrobot system, the microrobot structure was considered to be isotropic in the $\mathrm{x}$ and $\mathrm{z}$ directions with two degrees of freedom (DOFs) ( $D_{y x}$ deformation in the yx plane in the x direction, and $D_{y z}$ deformation in the yz plane in the $\mathrm{z}$ direction). The deformations can be written as:

$$
\begin{aligned}
& {\left[D_{y z}\right]_{3 \times 1}=\sum_{i=1}^{n} \beta_{i} \vec{\tau}_{i x}=\sum_{i-1}^{n} \alpha_{i} \vec{f}_{i z}} \\
& {\left[D_{x z}\right]_{3 \times 1}=\sum_{i=1}^{n} \beta_{i} \vec{\tau}_{i z}=\sum_{i-1}^{n} \alpha_{i} \vec{f}_{i x}}
\end{aligned}
$$

The $\alpha$ and $\beta$ terms reflect the mechanical properties (elasticity, shear modulus) and structural geometry that map the forces and torques to the deformations, and $n$ is the number of permanent magnets in the microrobot. Now, considering Equation (9), and using Equation (8), the relationship between the magnetic field and deformation can be written as:

$$
\begin{aligned}
& {\left[D_{y z}\right]_{3 \times 1}=\sum_{i=1}^{n}\left[\begin{array}{cccccccc}
0 & -m_{z i} & m_{y i} & m_{x i} & m_{y i} & m_{z i} & 0 & 0 \\
m_{z i} & 0 & -m_{x i} & 0 & m_{x i} & 0 & m_{y i} & m_{z i} \\
-m_{y i} & m_{x i} & 0 & -m_{z i} & 0 & m_{x i} & -m_{z i} & m_{y i}
\end{array}\right]_{3 \times 8}[K]_{8 \times 8}\left[\begin{array}{l}
B \\
B_{g}
\end{array}\right]_{8 \times 1}} \\
& K_{8 \times 8}=\operatorname{diag}\left(\beta_{i}, \beta_{i}, \beta_{i}, \alpha_{i}, \alpha_{i}, \alpha_{i}, \alpha_{i}, \alpha_{i}\right)
\end{aligned}
$$

where $M_{\tau}$ packs the dipole terms contributing to magnetic torque. Combining Equations (6) and (7), the total force and torque matrix can be written as:
Figure 3 shows the geometry of the soft microrobot and the configuration used for steering. The magnetization vector depends on the orientation of the permanent magnets, which was

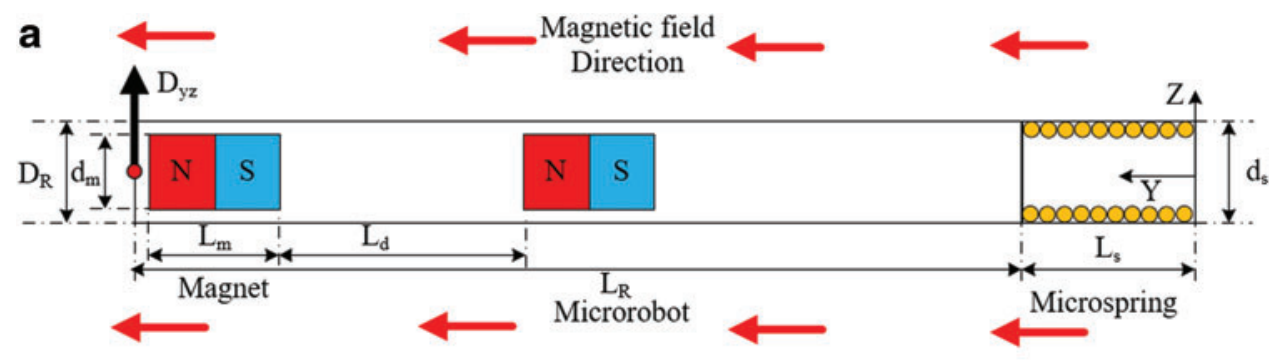

b

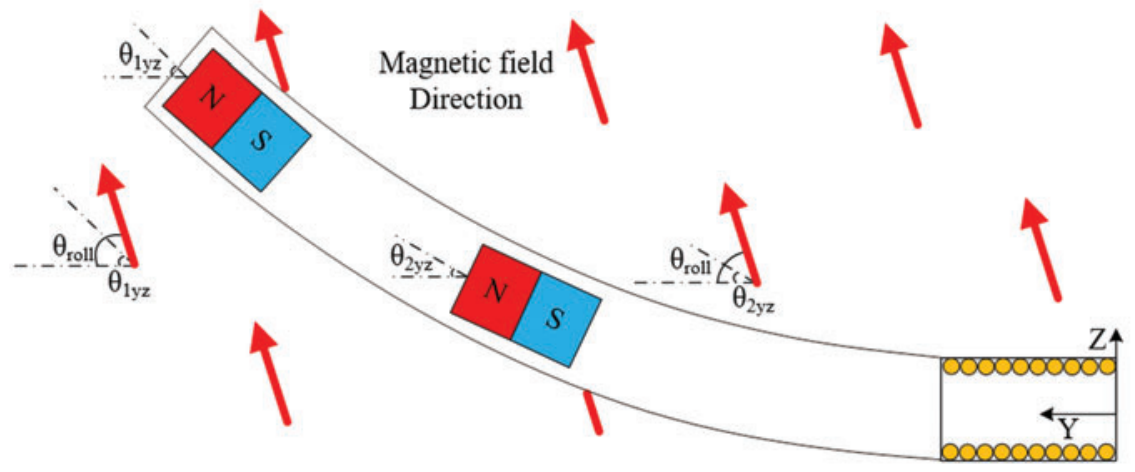

FIG. 3. Magnetic actuation of the microrobot to steer the guidewire. (a) The geometrical parameters of the microrobot in the initial position: $D_{R}$, microrobot diameter, $d_{m}$, magnet diameter, $L_{R}$, microrobot length, $L_{m}$, magnet length, and $L_{d}$, the distance between the magnets. (b) Microrobot deformation with a change in the magnetic field, $\theta_{\text {roll }}$ is the field direction, and $\theta_{1}$ and $\theta_{2}$ are the deformation angles of the first and second magnets, respectively. The angular differences between the magnets and the field are represented by $\theta_{1 y z}$ and $\theta_{2 y z}$. Color images are available online. 
initially chosen to be the y direction; thus: $m_{1}=m_{2}=m\left[\begin{array}{lll}0 & 1 & 0\end{array}\right]$. However, the direction of the magnetization vector changes as the position of the microrobot changes, as shown in Figure 3. The orientations of the magnets are represented by $\theta_{1 y z}, \theta_{2 y z}$, $\theta_{1 y x}$, and $\theta_{2 y x}$ (Fig. 3). Therefore, considering both the rotational angles and the directions of initial magnetization, the magnetization vector for each magnet $i$ can be written as:

$$
\left[\begin{array}{c}
m_{x i} \\
m_{y i} \\
m_{z i}
\end{array}\right]=\left[\begin{array}{ccc}
c \theta_{i y x} & -s \theta_{i y x} & 0 \\
c \theta_{i y z} s \theta_{i y x} & c \theta_{i y z} c \theta_{i y x} & -s \theta_{i y z} \\
s \theta_{i y z} s \theta_{i y x} & s \theta_{i y z} c \theta_{i y x} & c \theta_{i y z}
\end{array}\right]\left[\begin{array}{l}
0 \\
m \\
0
\end{array}\right]
$$

where $s$ and $c$ indicate $\sin$ and $\cos$, respectively. When steering the soft microrobot, the magnetic gradient is considered to be zero and magnetic torque (only) is used for steering. Consequently, considering Equation (12), Equation (11) can be written as:
Table 2. Material Properties of the Soft Microrobot Used in Mathematical Modeling AND FInITE Element ANALYSIS ${ }^{34,42,43}$

\begin{tabular}{lcc}
\hline Material & Polydimethylsiloxane & $\begin{array}{c}\text { Neodymium } \\
\text { magnet } \\
(\text { NdFeB, N52) }\end{array}$ \\
\hline $\begin{array}{l}\text { Density }\left(\mathrm{kg} / \mathrm{m}^{3}\right) \\
\begin{array}{l}\text { Young's } \\
\text { modulus }(\mathrm{Pa})\end{array}\end{array}$ & 0.97 & 7,500 \\
$\begin{array}{l}\text { Poisson ratio } \\
\text { Relative }\end{array}$ & 0.49 & $160 \times 10^{3}$ \\
$\quad$ permeability & 1 & 0.24 \\
$\begin{array}{l}\text { Remanence flux } \\
\text { density (T) }\end{array}$ & - & 1.05 \\
\hline
\end{tabular}

$$
\begin{aligned}
& {\left[D_{y z}\right]_{3 \times 1} \sum_{i=1}^{n}\left[\begin{array}{ccc}
0 & -m\left(s \theta_{i y z} c \theta_{i y x}\right) & -m\left(c \theta_{i y z} c \theta_{i y x}\right) \\
-m\left(s \theta_{i y z} c \theta_{i y x}\right) & 0 & -m\left(s \theta_{i y x}\right) \\
-m\left(c \theta_{i y z} c \theta_{i y x}\right) & -m\left(s \theta_{i y x}\right) & 0
\end{array}\right]_{3 \times 3} \operatorname{diag}\left(\beta_{i}, \beta_{i}, \beta_{i}\right)\left[\begin{array}{c}
\beta_{x} \\
\beta_{y} \\
\beta_{z}
\end{array}\right]} \\
& {[D]_{3 \times 1}\left(\sum_{i=1}^{n}\left[M_{i}\right]_{3 \times 3}\left[\beta_{i}\right]_{3 \times 3}\right)[B]_{3 \times 1}}
\end{aligned}
$$

In Equation (13), the matrix $M_{i}$ is the magnetization vector for each magnet, $\beta_{i}$ maps the magnetic torque to the deformation, and the matrix $B$ is the magnetic field. To steer the soft microrobot, we consider the field to be constant in the $y$ direction $[0 \mathrm{~B} 0]^{\mathrm{T}}$ and change the field direction to generate the magnetic torque according to Equation (13). Therefore, deformation can be described as:

$$
[D]_{3 \times 1}\left(\sum_{i=1}^{n}\left[M_{i}\right]_{3 \times 3}\left[B_{i}\right]_{3 \times 3}\right)\left[\begin{array}{ccc}
1 & 0 & 0 \\
0 & c \theta_{\text {roll }} & -s \theta_{\text {roll }} \\
0 & s \theta_{\text {roll }} & c \theta_{\text {roll }}
\end{array}\right]\left[\begin{array}{ccc}
c \theta_{\text {yaw }} & -s \theta_{\text {yaw }} & 0 \\
s \theta_{\text {yaw }} & c \theta_{\text {yaw }} & 0 \\
0 & 0 & 1
\end{array}\right]\left[\begin{array}{c}
0 \\
B \\
0
\end{array}\right]
$$

where $s$ and $c$ indicate $\sin$ and $\cos$, respectively, $B$ is the constant magnetic field, and $\theta_{\text {roll }}$ and $\theta_{\text {yaw }}$ are the roll and yaw angles about the $x$ and $z$ axes, respectively. Finally, Equation (14) can be packed as:

$$
[D]_{3 \times 1}\left(\sum_{i=1}^{n}\left[\left[M_{i}\right]_{3 \times 3}\left[B_{i}\right]_{3 \times 3}\right]\right)\left[R_{\text {roll }}\right]_{3 \times 3}\left[R_{y a w}\right]_{3 \times 3}[B]_{3 \times 1}
$$

where $R_{\text {roll }}$ and $R_{\text {yaw }}$ are the rotational matrices about the $x$ and $z$ axes, $B$ is the constant magnetic field in the $y$ direction, the matrix $M_{i}$ is the magnetization vector for each magnet,

Table 1. Geometrical Information Used IN THE FABRication and Finite ElEMENT ANALysis Simulation of THE Soft Microrobot

\begin{tabular}{lccccc}
\hline & \multicolumn{2}{c}{ Diameter } & & \multicolumn{2}{c}{ Length } \\
\cline { 2 - 3 } \cline { 5 - 6 } & \multicolumn{2}{c}{ Parameter } & Value $(\mu \mathrm{m})$ & Parameter & Value $(\mu m)$ \\
\hline Microrobot & $\mathrm{D}_{\mathrm{R}}$ & 500 & & $\mathrm{~L}_{\mathrm{R}}$ & 3,800 \\
Magnet & $\mathrm{d}_{\mathrm{m}}$ & 400 & & $\mathrm{~L}_{\mathrm{m}}$ & 800 \\
Microspring & $\mathrm{d}_{\mathrm{s}}$ & 500 & & $\mathrm{~L}_{\mathrm{s}}$ & 2000 \\
Guidewire & $\mathrm{d}_{\mathrm{g}}$ & 360 & & $\mathrm{~L}_{\mathrm{g}}$ & $1.9 \times 10^{6}$ \\
\hline
\end{tabular}

and $\beta_{i}$ maps the magnetic torque to the deformations. Equation (15) thus yields the deformation. However, the matrix $M$ is a function of the magnitude of magnetization $(m)$ and the magnet configurations $\left(\theta_{1 y z}, \theta_{2 y z}, \theta_{1 y x}\right.$, and $\left.\theta_{2 y x}\right)$; and $\beta_{i}$ depends on the mechanical properties and structural geometry of the microrobot. Structural nonlinearity limits the mathematical modeling; thus, FEA was used to determine the deformations.

\section{The FEA model for the soft microrobot}

To simplify the model, the soft microrobot was assumed to be isotropic and the model was derived in 2D. Deformation of the soft microrobot by an external magnetic field was studied by using the COMSOL Multiphysics ${ }^{\circledR}$ suite v. 5.3. (COMSOL AB, Stockholm, Sweden). The microrobot geometry is shown in Figure 3, and the detailed geometrical parameters are listed in Table 1. As shown in Figure 3, the soft microrobot consists of two permanent magnets and a PDMS cantilever beam. The material properties and simulation conditions are given in Tables 2 and 3 , respectively. The microrobot was modeled by using the

Table 3. Experimental and Simulation Conditions For Steering Mediated by the Soft Microrobot

Parameter Value

Direction of the magnetic field $\left(^{\circ}\right)$ From 30 to 170 Intensity of the magnetic field (mT)

\section{$5,10,15$}


geometrical information in Table 1, and a triangular element was used to generate the mesh.

The external field magnitude was considered to be a maximum of $15 \mathrm{mT}$, and the field direction was initially designed to align in the $y$ direction. The magnetic field di- rection $\left(\theta_{\text {roll }}\right)$ was varied between $10^{\circ}$ and $170^{\circ}$, and structural analysis was used to determine deformation with respect to the change in that direction. Figure 4 shows the deformations associated with various directions of the magnetic field. The results are presented and discussed in the Results section.
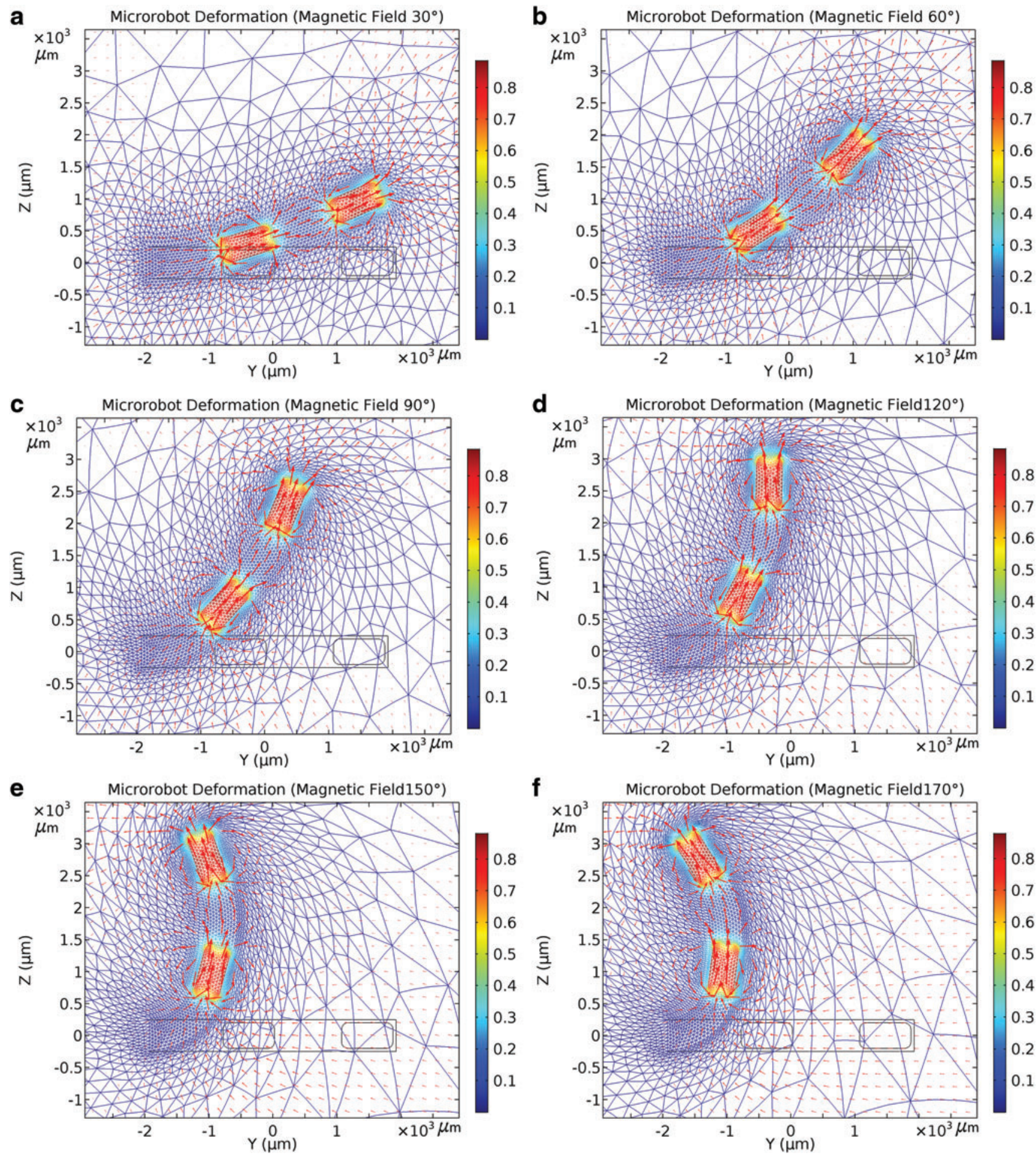

FIG. 4. Finite element analysis simulation in the COMSOL multiphysics environment. Deformations of the microrobot by an external magnetic field of intensity of $15 \mathrm{mT}$ at angles of (a) $30^{\circ}$, (b) $60^{\circ}$, (c) $90^{\circ}$, (d) $120^{\circ}$, (e) $150^{\circ}$, and (f) $170^{\circ}$. The right color bar shows the magnetic flux density $(\mathrm{T})$. Color images are available online. 


\section{Fabrication of the soft microrobot}

The microrobot must be of submillimeter scale, exhibit high deformability and steerability. The micrometer scale enables the guidewire with the soft microrobot (GSM) to be inserted into coronary arteries. High-level deformability enables guidance over a wide range of branch angles, and high steerability affords precise control. Therefore, we fabricated the microrobot from PDMS and incorporated two permanent magnets (NdFeB, N52; Ningbo Zhonghang Magnetic Materials Co., Ltd, Zhejiang, China). The microrobot is cylindrical in shape (thus symmetrical), affording multi-axis steerability. The microrobot geometry and material properties are listed in Tables 1 and 2, respectively.

A metal master, commonly used for replica molding, served as the master for PDMS molding. ${ }^{34,35}$ The metal master was made from stainless steel and was manufactured by wire electrical discharge machining; thus, it can be reused multiple times. We first prepared a replica PDMS mold (a PDMS master) by using a metal master. A mixture of PDMS prepolymer and the curing agent was poured into the metal master, and the replica PDMS mold was obtained by peeling the metal master away. The next step was re-molding by using a PDMS master; this second PDMS mold was used to fabricate the soft microrobot. PDMS is optically transparent, which aids fabrication (as shown in Fig. 5). As the mold and beam of the microrobot are made of the same material (PDMS), they tend to stick together after the beam is cured. We, thus, hydrophobically coated the mold and the beam by using an oxygen plasma system (Cute; FEMTO SCIENCE, Seoul, Korea) and trichloro(1H,1H,2H,2H-perfluorooclyl)silane (Sigma-Aldrich, St. Louis, MO). Thin layers of perfluorooctyl trichlorosilane were deposited on the PDMS surfaces in a closed chamber under a pressure of 0.5 bar at $80^{\circ} \mathrm{C}$ for $2 \mathrm{~h} .{ }^{36}$ This improved detachment of the beam from the mold.

The fabrication process is shown in Figure 5. Initially, the PDMS mold was filled with a Sylgard 184 silicone elastomer mixture (Dow Corning Corp., Midland, MI) at a base:curing
FIG. 5. Fabrication of the soft microrobot composed of a beam (polydimethylsiloxane [PDMS] silicone and a neodymium magnet), a connection (a microspring), and a guidewire. (a) The PDMS metal master mold and replica molding using PDMS; (b) pouring of the silicone elastomer mixture into the metal PDMS master mold, and curing; (c) alignment of the two-magnet, 1-mm-diameter rod-shaped PDMS block and the microspring; (d) pouring of the silicone elastomer mixture into the PDMS master mold, and curing; (e) adjusting the length of the microrobot; and (f) connecting the microrobot to the guidewire to allow guidewire steering. Color images are available online.
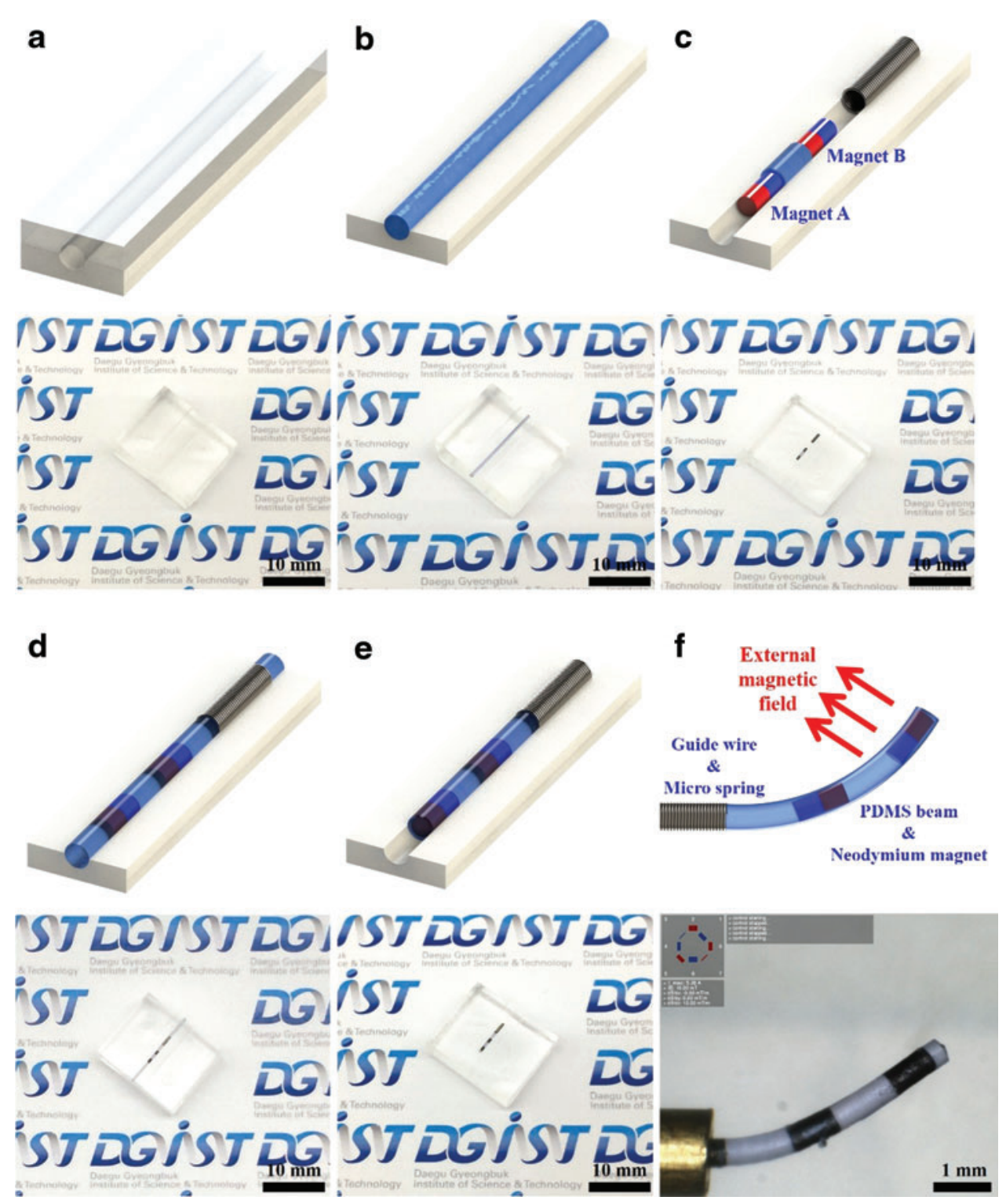
agent weight ratio of $10: 1$ by using methyl blue to visualize the elastomer mixture inside the mold, and the mixture was then cured at $80^{\circ} \mathrm{C}$ for $8 \mathrm{~h}^{36}$ (Fig. 5b). A rod-shaped PDMS beam $(1 \mathrm{~mm})$ was used in the next fabrication step. The beam, permanent magnets, and microspring were aligned on the mold, as shown in Figure 5c. The microspring was used to connect the PDMS beam to a guidewire. The distance between the magnet B and the microspring was $1 \mathrm{~mm}$. As shown in Figure 5d, the silicone elastomer mixture was then used to fill the mold in the closed chamber under vacuum followed by curing at $80^{\circ} \mathrm{C}$ for $8 \mathrm{~h}$. The final structure is shown in Figure 5e. Finally, the microrobot was connected to a conventional guidewire (Fig. 5f). In this approach, a conventional commercially available guidewire was used for navigation. This guidewire is in clinical use, so its application does not raise any toxicity issues. The permanent magnets in the microrobot are enclosed within biocompatible PDMS material. The biocompatibility test results for the PDMS material revealed no toxicity (Supplementary Fig. S2). Therefore, the microrobot is safe for future use in in-vivo studies. ${ }^{37}$

\section{Experimental setup}

The OctoMag system precisely changes the direction of the magnetic field. ${ }^{12}$ Rectilinear motion of the GSM was controlled by a master/slave system ${ }^{33}$ (Fig. 6). The OctoMag system features eight Helmholtz/Maxwell electromagnetic coils that are capable of generating a maximum magnetic field of $120 \mathrm{mT}$ in a region of interest (a sphere $8 \mathrm{~cm}$ in radius). The OctoMag system afforded five DOFs to untethered microrobots (three position DOFs, two orientation DOFs) ${ }^{38-40}$ The system can also control a soft microrobot (with two DOFs) by changing the direction of the magnetic field.
The diameter of the guidewire (Hi-Torque Powerturn hydrophilically coated guidewire; Abbott Vascular, Santa Clara, CA) connected to the microrobot is $0.36 \mathrm{~mm}$, and its length is $190 \mathrm{~cm}$. The guiding catheter (Cordis, Miami Lakes, FL) is a flexible thin plastic tube that is $1.67 \mathrm{~mm}$ in diameter.

For the in vitro experiments, a 2D blood vessel phantom was fabricated by a 3D printer (ProJet MJP 3500; 3D Systems, Rock Hill, SC) as shown in Figure 6 (top left). Both the width and the depth of the 2D phantom were $2 \mathrm{~mm}$, and the branch angles varied from $30^{\circ}$ to $120^{\circ}$ to mimic the coronary arteries in a $2 \mathrm{D}$ environment. The 3D blood vessel phantom (Elastrat, Geneva, Switzerland) made of soft silicone mimics the aortic arch and the left and right coronary arteries, including the marginal and posterior intraventricular arteries of the latter artery. The left coronary artery includes the left anterior intraventricular and circumflex arteries. ${ }^{41}$

\section{Results}

\section{Model analysis and feedforward angular steering}

In the FEA simulation, the magnetic field directions, as shown in Figure 4, varied from $30^{\circ}$ to $170^{\circ}$ and the magnetic torque rotated the PDMS cantilever beam. Figure 4 shows the simulated (COMSOL) deformations of the soft microrobot caused by the external magnetic field. To have a condition similar to the model in the experiments, the microrobot was fixed at the end of a rigid holder. The experimental results with the guidewire and a rigid holder revealed negligible variation, $4 \%$ on average (Supplementary Fig. S1). The red arrows indicate the strength and direction of the magnetic field (the vector sums of the magnetic fields of the OctoMag and the two magnets). At regions distant from the two magnets, the field is influenced principally by the magnetic steering system (the external magnetic field) but, in regions

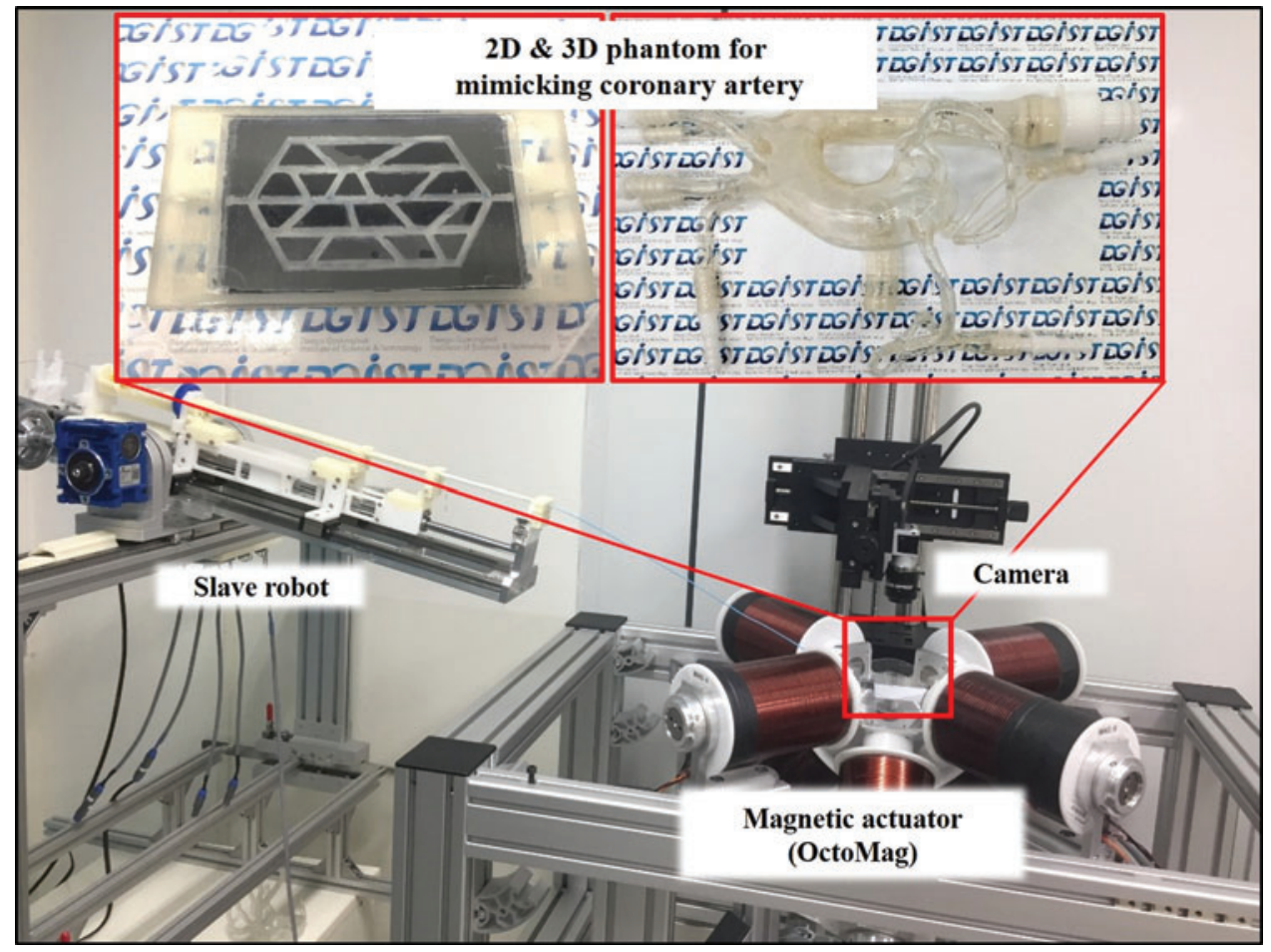

FIG. 6. The magnetic actuator (OctoMag), master/slave system, two-dimensional (2D) and three-dimensional (3D) blood vessel phantoms, and the charge-coupled device camera. The soft microrobot steered the guidewire in 2D and 3D experiments. Color images are available online. 
close to the magnets, the field generated by those magnets is dominant. Initially, the magnets and the external field point in the same direction (Fig. 4a); however, structural limitations gradually render the magnets unable to fully follow the steering magnetic field. Figures $4 \mathrm{a}-\mathrm{f}$ confirm that the flexible microrobot is deformed by the external magnetic field and that the deformation angle increases as the change from the original direction of the external magnetic field increases. Figure $4 \mathrm{f}$ shows that, at an external field direction of $170^{\circ}$, structural limitations render the microrobot unable to assume the same direction.

The measured and simulated deformation angles are compared in Figure 7a. The deformation angles change as the external magnetic field direction $\left(R_{\text {roll }}\right)$ varies from $30^{\circ}$ to $170^{\circ}$ at magnetic field intensities of 5,10 , and $15 \mathrm{mT}$. The maximum deformation angle yielded by the simula-
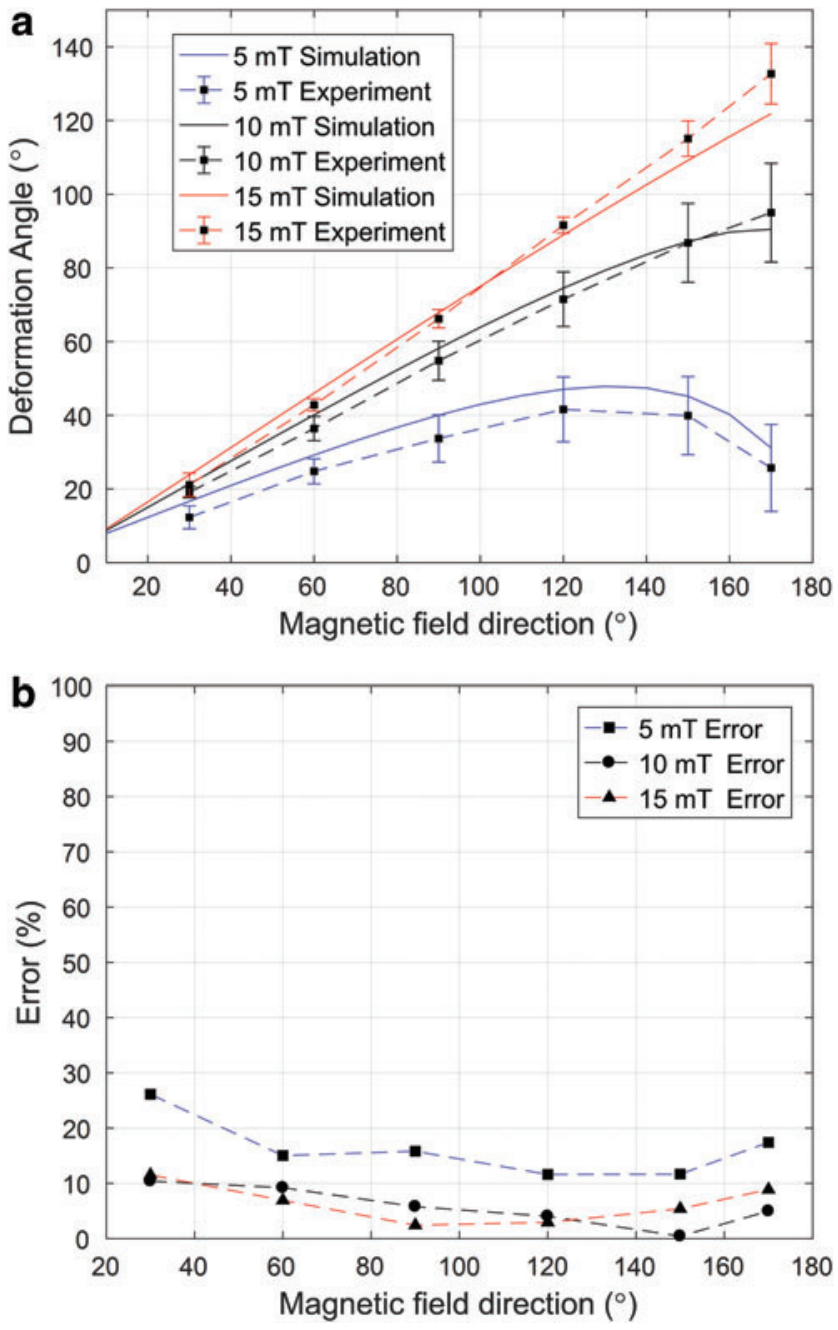

FIG. 7. Simulated and experimental deformation angles when the field directions varied $(n=3$ for the experimental results). (a) Simulation (field directions between $10^{\circ}$ and $170^{\circ}$ ) and experimental (field directions $30^{\circ}, 60^{\circ}, 90^{\circ}, 120^{\circ}$, $150^{\circ}$, and $170^{\circ}$ ) results. (b) The differences between the experimental results and the simulations for field directions of $30^{\circ}, 60^{\circ}, 90^{\circ}, 120^{\circ}, 150^{\circ}$, and $170^{\circ}$. Color images are available online. tion was $121.8^{\circ}$ when the direction of the external magnetic field $(15 \mathrm{mT})$ was $170^{\circ}$; the measured, maximum, experimental deformation angle was $132.7^{\circ}$ under these conditions.

Figure $7 \mathrm{~b}$ shows the percentage differences between simulations and the experiments. All errors were $<20 \%$, showing that the simulations and experimental data agreed, with the exception of the results obtained by using a field of magnetic intensity of $5 \mathrm{mT}$ at $30^{\circ}$.

\section{Soft microrobot trajectory tracking in the $2 D$ phantom}

The main function of the developed soft microrobot is to navigate a conventional guidewire in multi-blood-vessel branches. We tested trajectory tracking in vitro in a waterfilled 2D phantom placed in the region of interest of the OctoMag. Microrobot rotation was controlled by changing the direction of the magnetic field, and rectilinear motion was controlled by the master/slave system. A trajectory planning algorithm was developed to enhance the human operator navigation capabilities. The trajectory information consists of the vessel length (rectilinear displacement) and the vessel angle in the bifurcations (rotational displacement) extracted and used to estimate rectilinear and rotational displacements.

The delegated task of GSM navigation is a semiautomatic task. Modeling is used to predict the linear and rotational displacements, and an operator guarantees the needed high precision. The flowchart in Figure 8 shows the trajectory tracking process (the OctoMag, the master/ slave system, and the optical camera). As the flowchart indicates, vessel data (length, diameter, and direction) were initially calculated by using the MATLAB software (MathWorks, Inc., Natick, MA). When a $20 \mathrm{~mm} / \mathrm{s}$ push velocity was demanded by the master system, the scaling between the master and slave (200-11.4 mm) afforded a rectilinear velocity of $1.14 \mathrm{~mm} / \mathrm{s}$. The time required for rotational displacement was assumed to be $5 \mathrm{~s}$. Based on this information, we generated the path shown in Figure 9a. This is a displacement-time diagram showing how to attain the target position using a predefined path. Rectilinear motion continues until the robot reaches the first bifurcation. Then, the direction of the magnetic field is changed based on the model developed in the previous section. Although the algorithm can predict linear and angular displacements with the developed models, the operator has complementary skills and can adjust the linear and angular displacements via master-slave and magnetic-field control systems. This process continues until the robot attains the desired position.

The guidewire trajectory is shown in Figure 9b (dotted line). The GSM is initially driven by the master/slave system. Once the GSM reaches a bifurcation (Fig. 9c) and must turn $60^{\circ}$ counterclockwise (ccw), it is steered $80^{\circ} \mathrm{ccw}$ by the external magnetic field. Figure $9 \mathrm{~d}$ shows the guidewire advancing along the planned trajectory and magnetic steering at the second branch (Fig. 9e; $60^{\circ}$ clockwise [cw]). The GSM is controlled similarly in Figure 9f. Finally, the GSM attains the target (Fig. 9g). The Supplementary Video S1 shows the entire course of tracking inside the 2D phantom. The timedisplacement diagram during 2D tracking is also shown in Figure 9a. 


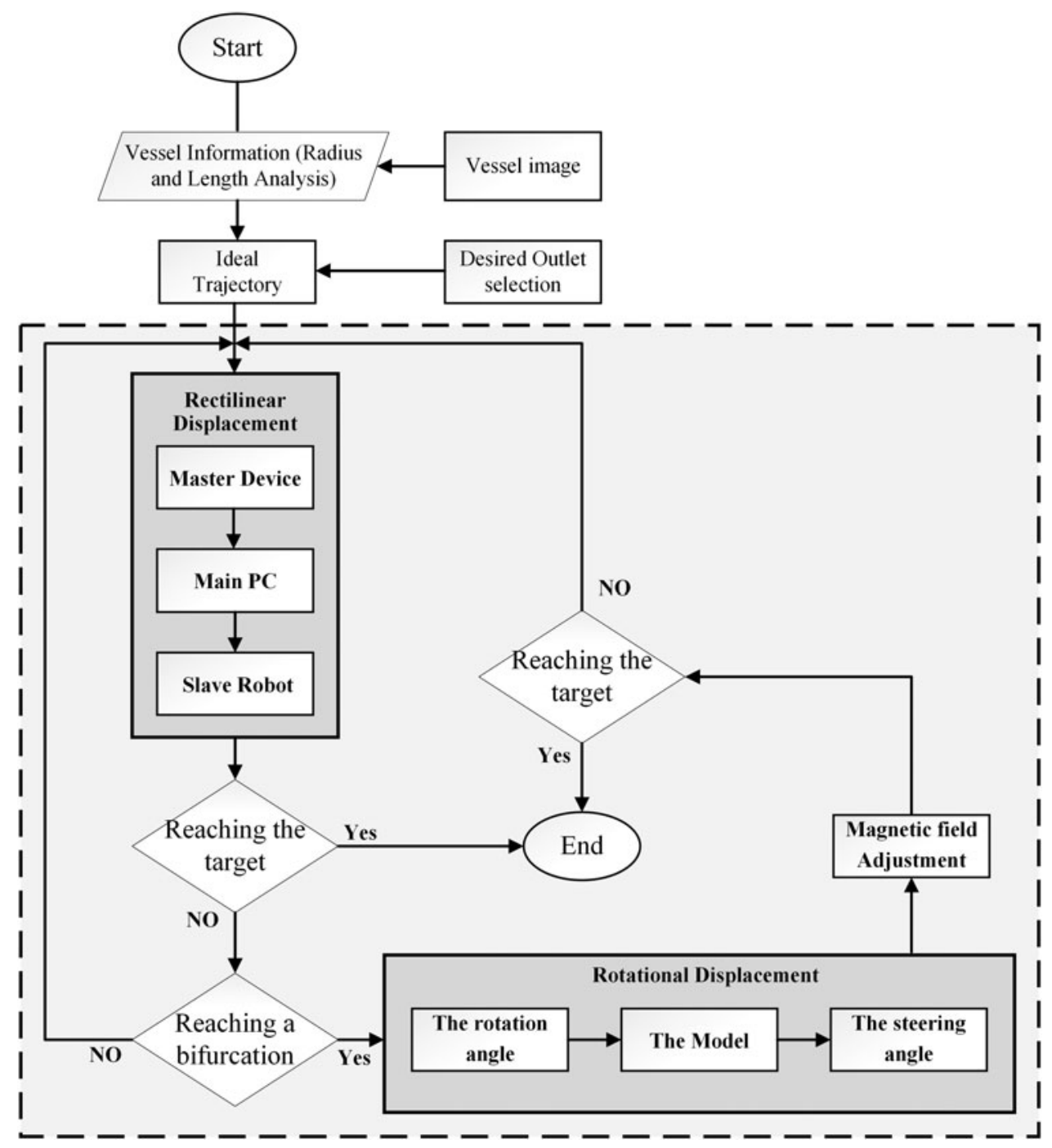

FIG. 8. Trajectory planning flowchart for 2D tracking.

\section{Trajectory tracking in the 3D phantom}

In the previous section, we used a $2 \mathrm{D}$ phantom. As the heart is a 3D structure, the microrobot must be controlled in 3D. Consequently, a transparent 3D phantom mimicking the coronary artery was used in a steering/ tracking test within three branches of the left coronary artery (Fig. 10 and Supplementary Video S1). Given that the geometry of the 3D phantom was known, only the top camera was used for tracking. In future studies, a bi-plane $\mathrm{X}$-ray system (two X-ray systems in a perpendicular orientation) will be used to register the patient's 3D CT image, enabling 3D locomotion in real time. The GSM is controlled by both the external magnetic field and the master/slave system.

The GSM was precisely steered, using the magnetic field (Fig. 10a), into each of the three branches as directed. To deliver the GSM into the right branch, deformations of $-30^{\circ}$ in pitch and $30^{\circ}$ in yaw were required (Fig. 10b). When targeting the middle branch, the figures were $-10^{\circ}$ pitch and $100^{\circ}$ yaw (Fig. 10c. When targeting the left branch, the figures were $0^{\circ}$ pitch and $140^{\circ}$ yaw (Fig. 10d). In conclusion, the microrobot exhibited dexterous 3D movement.

\section{Discussion}

We expand next on our modeling and experimental findings:

1. Effects of magnetic intensity

2. Curvature of the deformation angle

3. Variations between the model and the experiments

4. Working conditions

\section{Effects of magnetic intensity}

The change in deformation angle as the magnetic field changes is given by Equation (13). The deformation depends on the matrix $M$ (representing magnet geometry), the matrix $\beta$ (representing the mechanical properties of the microrobot), and the matrix $B$ (representing magnetic field intensity and direction). As $\theta_{i y z}$ and $\theta_{i y x}$ of matrix $M$ change when the field direction changes, matrix $M$ is a function of field direction. This explains the curvature of the deformation diagram (Fig. 7a); the magnitude of magnetization $(\mathrm{m})$ is constant. The deformation varies, however, with changes in magnetic intensity (matrix $B$ ). A higher intensity increases curvature, which is associated with greater deformation. 

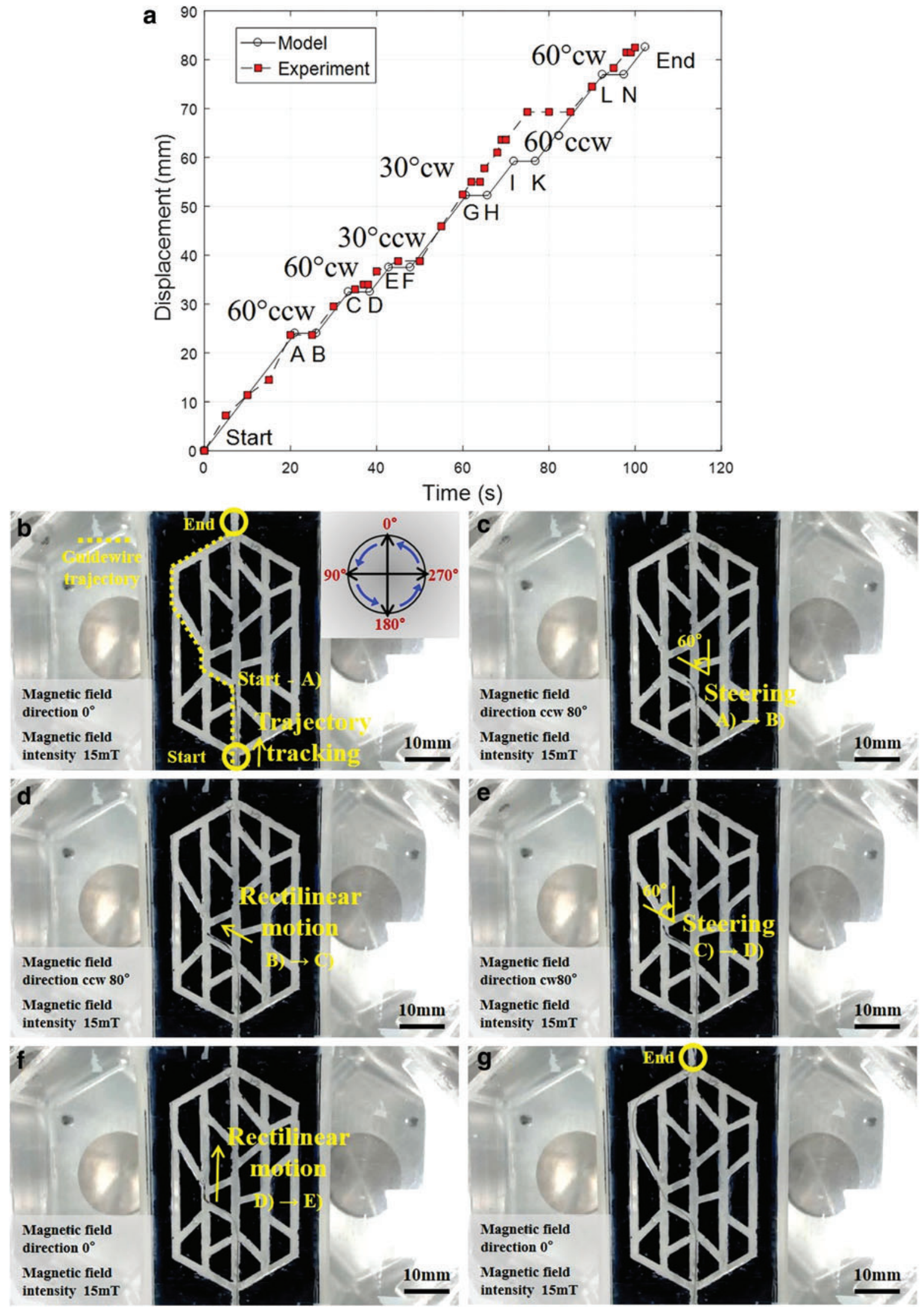

FIG. 9. In vitro tracking within a $2 \mathrm{D}$ blood vessel phantom with various branches and inclinations. (a) The timedisplacement trajectory for the desired path (the angles are the required deformation angles). (b)-(f) the steering and rectilinear motions of the soft microrobot and guidewire; and (g) attainment of the goal position. cw, clockwise; ccw, counterclockwise. Color images are available online. 

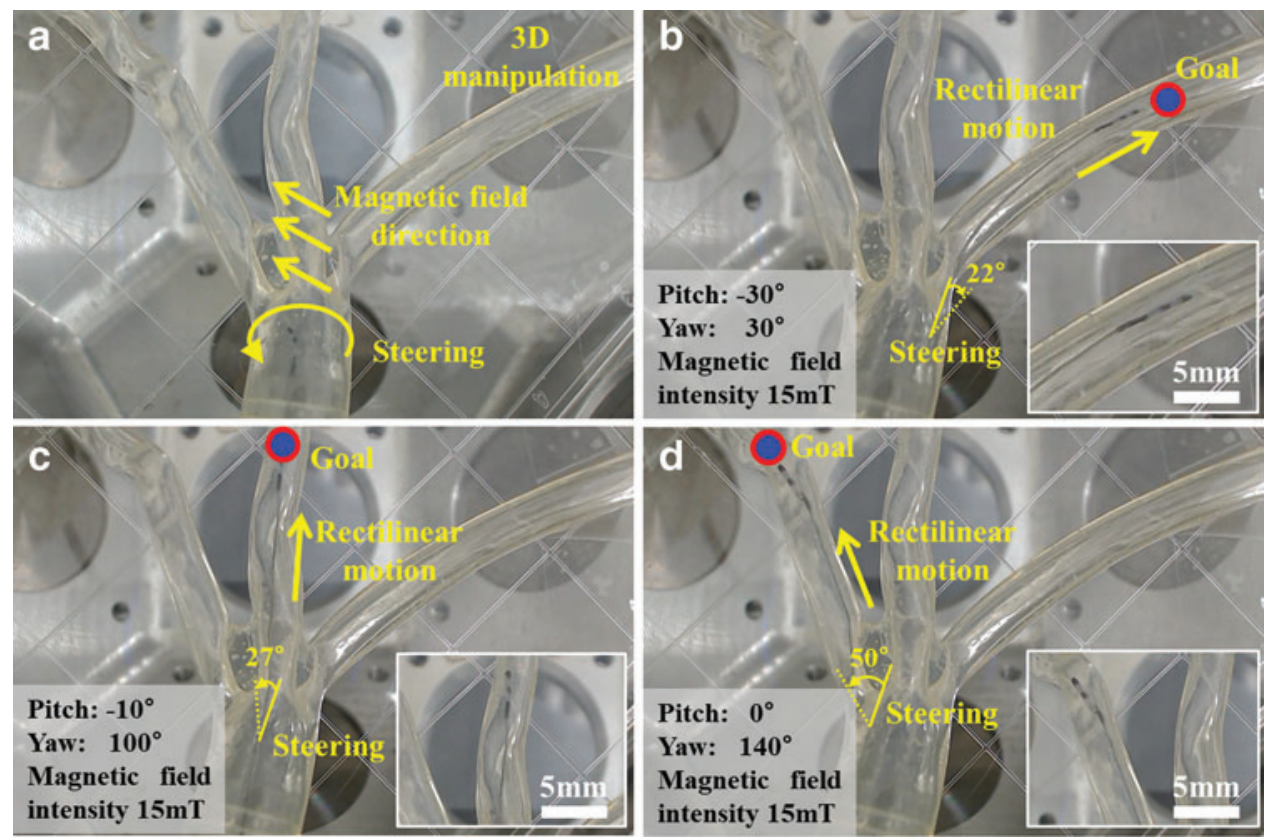

FIG. 10. In vitro tracking within a 3D phantom of a left coronary artery with three branches. (a) 3D steering of the soft microrobot. (b-d) $\mathrm{cw} 22^{\circ}$, ccw $27^{\circ}$, and ccw $50^{\circ}$ steering using the OctoMag to impart rectilinear motion via the master/slave system. Color images are available online.
For the field intensities of 5 and $10 \mathrm{mT}$, the maximum average deformation angles achieved in the experiments were $41.6^{\circ}$ and $95^{\circ}$, respectively. To achieve higher steerability, the field intensity was set to $15 \mathrm{mT}$, associated with a maximum average deformation angle of $132.7^{\circ}$ in the experiments.

\section{Curvature of the deformation angle}

The curvature of the deformation angle (both measured and simulated) was nonlinear (Fig. 7a). Initially, the microrobot deforms in a linear manner as the external field angle increases. However, once the field direction attains $130^{\circ}$ (for a 5-mT field) or $160^{\circ}$ (for a $10-\mathrm{mT}$ field), continued changes in field direction reverse the effects of angular deformation. The extent of deformation [as shown in Eq. (13)] depends on the magnetic torque (the force is considered to be zero). The value of torque [Eq. (4)] depends on the external magnetic intensity $(|B|)$, the magnetization vector $(|M|)$, and the sine of the angle between the two vectors $(\theta)$ (schematic of Fig. 11a). As both the magnetic field intensity and the magnetization vector are constant, the torque declines when $\theta$ passes through $90^{\circ}$, reducing the deformation angle.

The difference in curvature is represented by Equation (14). In the $y z$ plane, $i=2, \theta_{\text {yaw }}=0$, and $\beta_{1}=\beta_{2}=\beta$; Equation (14) can then be simplified to: $\operatorname{diag}(m \beta B)$, a normalized curvature factor $(N C F)$ is derived $[N C F=C F / \max (C F)]$ (Fig. 11a).

The $N C F$ for a field of intensity $5 \mathrm{mT}$ declines at $\theta_{\text {roll }}$ $>130^{\circ}$, reducing the magnetic torque. At a field intensity of 10 $\mathrm{mT}$, the $N C F$ peaks at $160^{\circ}$ and declines slightly thereafter. Finally, at a field intensity of $15 \mathrm{mT}$, the $N C F$ does not peak, and instead increases continuously. Figure 11a, b explains the curvature shown in Figure $7 \mathrm{a}$.

\section{Variation between the experimental data and simulations}

With the exception of the $30^{\circ}$ direction, the model followed the experimental results closely. For the $30^{\circ}$ condition, the magnitude of the deviation between the experimental and simulation results for 10 and $15 \mathrm{mT}$ was within the ranges of acceptable deviation (10\% and $11 \%$, respectively). For the 5-mT condition, the model and experimental curvatures were similar, and the magnitude of the deviation between the model and experiments changed only slightly (Fig. 7). Nevertheless, because the magnitude of the deformation in the model was low (Fig. 7) in error calculations, a maximum error of $26.1 \%$ was observed at $30^{\circ}$.

$$
C F=\left[\begin{array}{ccc}
0 & -\left(s \theta_{1 y z}+s \theta_{2 y z}\right) & c \theta_{1 y z}+c \theta_{2 y z} \\
s \theta_{1 y z}+s \theta_{2 y z} & 0 & 0 \\
c \theta_{1 y z}+c \theta_{2 y z} & 0 & 0
\end{array}\right] \operatorname{diag}(m \beta B)_{3 \times 3}\left[\begin{array}{l}
0 \\
c \theta_{\text {roll }} \\
s \theta_{\text {roll }}
\end{array}\right]
$$

where $C F$ is the curvature factor. The curvature factor $C F$ depends on $\theta_{1 y z}, \theta_{2 y z}$, and $\theta_{\text {roll }} . \theta_{1 y z}$ and $\theta_{2 y z}$ are calculated by using the deformation angles of the model (Fig. 7a) and $\theta_{\text {roll }}$ ranges between $10^{\circ}$ and $170^{\circ}$. To eliminate the effects of

\section{Working conditions}

A high steering angle was achievable in a magnetic field of $15 \mathrm{mT}$. Linear behavior was observed both experimentally 


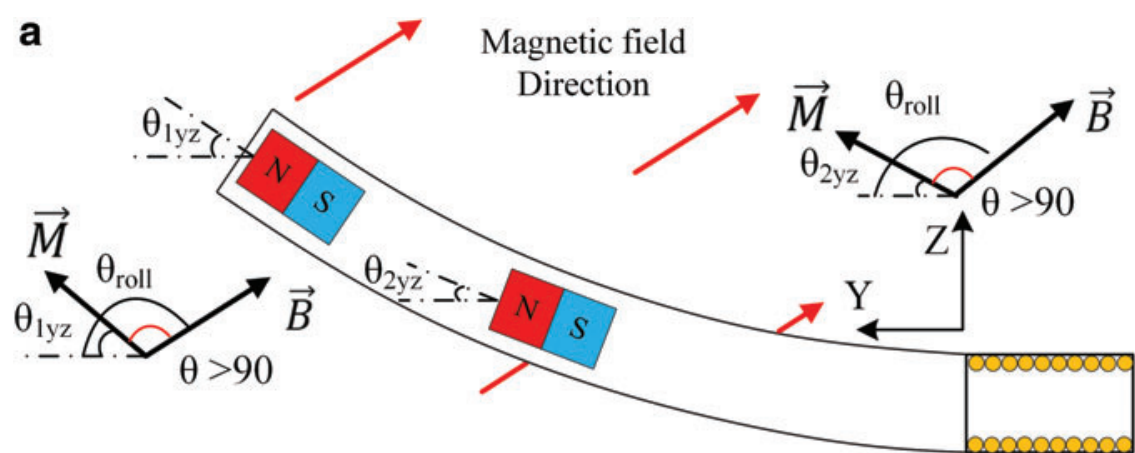

FIG. 11. (a) Schematic of microrobot deformation by magnetic torque when $\theta>90$. (b) Simulation of the normalized curvature factor by variation in magnetic field direction. Color images are available online.

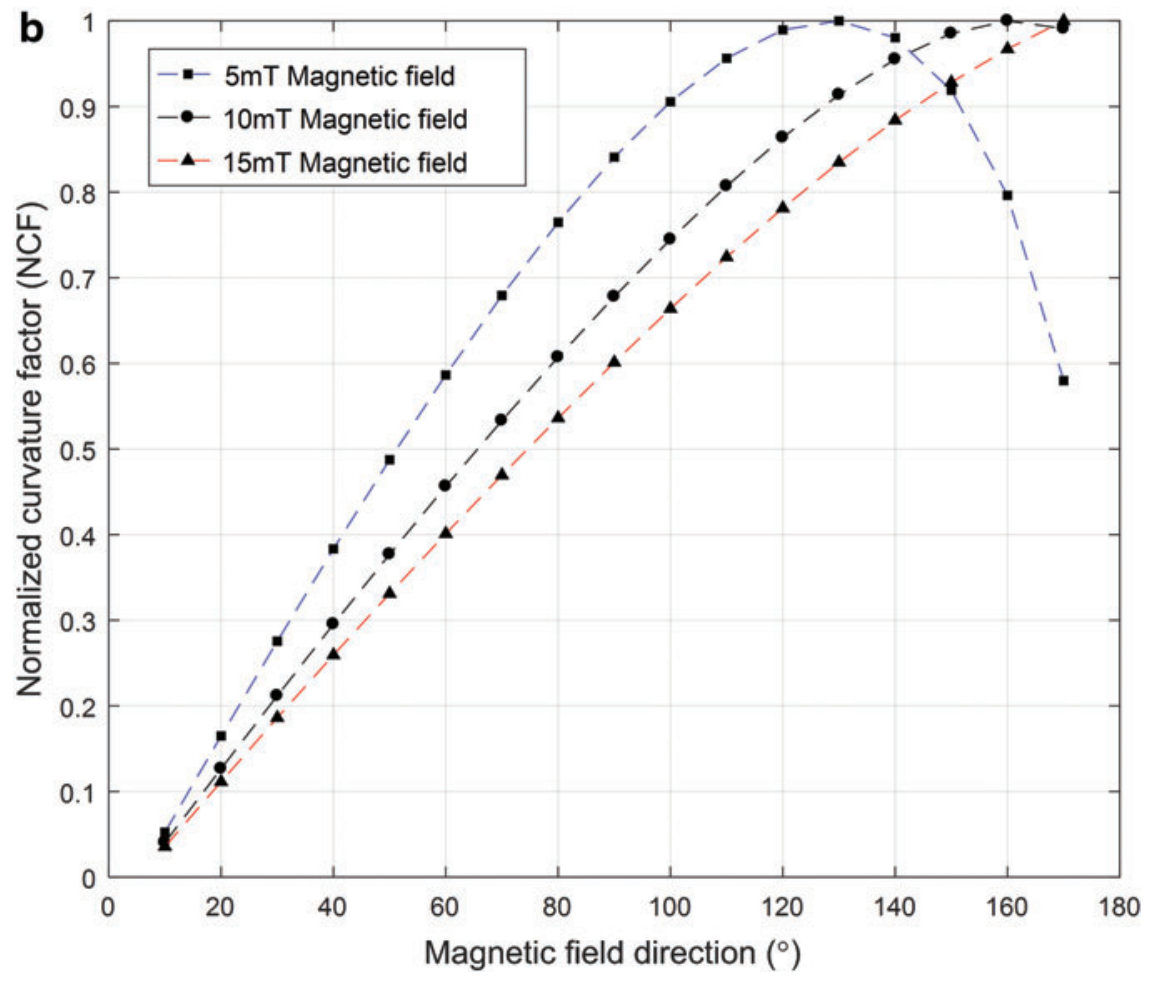

and in simulations. To avoid possible microrobot buckling, we suggest that the magnetic field direction be held within $0-150^{\circ}$. The error is then $11.5 \%$, rendering the results more reliable.

\section{Conclusion}

We developed a soft microrobot improving intravascular guidewire steerability and a model for analysis of angular deformation. The microrobot, composed of PDMS, a microspring, and two permanent magnets, was fabricated by casting using a PDMS mold. Our mathematical model successfully estimated microrobot deformation; the experimental data and the simulations were in good agreement. The deformation angle depends on both the direction and intensity of the magnetic field. The intensity is held constant, and the field direction is used for steering. The experimental deformation angle ranged from $21.1^{\circ}$ to $132.7^{\circ}$ at a magnetic field intensity of $15 \mathrm{mT}$. Therefore, the soft microrobot could be steered inside a complex $2 \mathrm{D}$ phantom. To explore possible applications in terms of PCI, we performed in vitro steering and tracking tests inside a water-filled 2D phantom mim- icking the coronary arteries. The microrobot engaged in rectilinear motion and traced a complex path controlled by the direction of the magnetic field. Similarly, a 3D phantom of the left coronary arteries was used to test 3D tracking performance. The microrobot was successfully guided into any desired arterial branch. Thus, our system improves guidewire steerability and will find applications in robotassisted PCI procedures. The Supplementary Video S1 shows successful 2D and 3D tracking by the soft microrobot. The results presented in this work show a microrobotic approach for improving conventional guidewire navigation. In the future, the developed approach can be used with a conventional catheter for angioplasty intervention. It also can be used with catheters equipped with novel magnetically actuated devices. Further, because perforation is of great concern in angioplasty procedures, the shape of the soft microrobotic tip should be considered in future studies.

\section{Acknowledgments}

Funding for this research was provided by the Korea Evaluation Institute of Industrial Technology (KEIT) funded 
by the Ministry of Trade, Industry \& Energy (No. 10052980) and the Global Research Laboratory from the National Research Foundation of Korea (NRF) funded by the Ministry of Science and ICT (No. NRF 2017K1A1A2013237).

\section{Author Disclosure Statement}

No competing financial interests exist.

\section{References}

1. Yim S, Gultepe E, Gracias DH, et al. Biopsy using a magnetic capsule endoscope carrying, releasing, and retrieving untethered microgrippers. IEEE Trans Biomed Eng 2014;61:513-521.

2. Gultepe E, Randhawa JS, Kadam S, et al. Biopsy with thermally-responsive untethered microtools. Adv Mater 2013;25:514-519.

3. Egolf PW, Shamsudhin N, Pané S, et al. Hyperthermia with rotating magnetic nanowires inducing heat into tumor by fluid friction. J Appl Phys 2016;120:064304.

4. Kim S, Lee S, Lee J, et al. Fabrication and manipulation of ciliary microrobots with non-reciprocal magnetic actuation. Sci Rep 2016;6:30713.

5. Kim S, Qiu F, Kim S, et al. Fabrication and characterization of magnetic microrobots for three-dimensional cell culture and targeted transportation. Adv Mater 2013;25: 5863-5868.

6. Hoshiar AK, Le T-A, Amin FU, et al. Studies of aggregated nanoparticles steering during magnetic-guided drug delivery in the blood vessels. J Magn Magn Mater 2017;427: 181-187.

7. Amin FU, Hoshiar AK, Do TD, et al. Osmotin-loaded magnetic nanoparticles with electromagnetic guidance for the treatment of alzheimer's disease. Nanoscale 2017;9: 10619.

8. Hoshiar AK, Le T-A, Amin FU, et al. A novel magnetic actuation scheme to disaggregate nanoparticles and enhance passage across the blood-brain barrier. Nanomaterials $2018 ; 8: 3$.

9. Zhang H, Hutmacher DW, Chollet F, et al. Microrobotics and mems-based fabrication techniques for scaffold-based tissue engineering. Macromol Biosci 2005;5:477-489.

10. Campuzano S, Orozco J, Kagan D, et al. Bacterial isolation by lectin-modified microengines. Nano Lett 2012;12: 396-401.

11. Balasubramanian S, Kagan D, Jack Hu CM, et al. Micromachine-enabled capture and isolation of cancer cells in complex media. Angew Chem Int Ed 2011;50: 4161-4164.

12. Kummer MP, Abbott JJ, Kratochvil BE, et al. OctoMag: An electromagnetic system for 5-dof wireless micromanipulation. In: IEEE International Conference on Robotics and Automation (ICRA); 2010, 3-7 May, pp. 1610-1616.

13. Ullrich F, Bergeles C, Pokki J, et al. Mobility experiments with microrobots for minimally invasive intraocular surgerymicrorobot experiments for intraocular surgery. Invest Ophthalmol Visual Sci 2013;54:2853-2863.

14. Nelson BJ, Kaliakatsos IK, Abbott JJ. Microrobots for minimally invasive medicine. Annu Rev Biomed Eng 2010; 12:55-85.

15. Charreyron SL, Zeydan B, Nelson BJ. Shared control of a magnetic microcatheter for vitreoretinal targeted drug delivery. In: IEEE International Conference on Robotics and Automation (ICRA); Singapore, Singapore; 2017, pp. 4843-4848.

16. Kearney K, Hira RS, Riley RF, et al. Update on the management of chronic total occlusions in coronary artery disease. Current atherosclerosis reports. 2017;19:19.

17. Benjamin EJ, Blaha MJ, Chiuve SE, et al. Heart disease and stroke statistics-2017 update: a report from the american heart association. Circulation 2017;135:146-603.

18. Touma G, Ramsay D, Weaver J. Chronic total occlusionscurrent techniques and future directions. IJC Heart Vasc 2015;7:28-39.

19. Jeong S, Choi $\mathrm{H}$, Go $\mathrm{G}$, et al. Penetration of an artificial arterial thromboembolism in a live animal using an intravascular therapeutic microrobot system. Med Eng Phys 2016;38:403-410.

20. Jeong S, Choi $\mathrm{H}$, Cha $\mathrm{K}$, et al. Enhanced locomotive and drilling microrobot using precessional and gradient magnetic field. Sens Actuators A Phys 2011;171:429-435.

21. Rucker DC, Jones BA, Webster RJ, 3rd. A geometrically exact model for externally loaded concentric-tube continuum robots. IEEE Trans Robot 2010;26:769-780.

22. Haga Y, Tanahashi Y, Esashi M. Small diameter active catheter using shape memory alloy. In: The Eleventh Annual International Workshop on Micro Electro Mechanical Systems. MEMS 98. Proceedings. Heidelberg, Germany; 1998, pp. 419-424.

23. Gul JZ, Yang YJ, Su KY, et al. Omni directional multimaterial soft cylindrical actuator and its application as a steerable catheter. Soft Robot 2017;4:224-240.

24. Muller L, Saeed M, Wilson MW, et al. Remote control catheter navigation: options for guidance under MRI. J Cardiovasc Magn Reson 2012;14:33.

25. Settecase F, Sussman MS, Wilson MW, et al. Magneticallyassisted remote control (MARC) steering of endovascular catheters for interventional MRI: a model for deflection and design implications. Med Phys 2007;34:3135-3142.

26. Chatzipirpiridis G, Erne P, Ergeneman O, et al. A magnetic force sensor on a catheter tip for minimally invasive surgery. In: Engineering in Medicine and Biology Society (EMBC), 37th Annual International Conference of the IEEE; 2015, pp. 7970-7973.

27. Sikorski J, Dawson I, Denasi A, et al. Introducing bigmaga novel system for $3 \mathrm{~d}$ magnetic actuation of flexible surgical manipulators. In: 2017 IEEE International Conference on Robotics and Automation (ICRA); 2017, pp. 3594-3599.

28. Back J, Lindenroth L, Rhode K, et al. Model-free position control for cardiac ablation catheter steering using electromagnetic position tracking and tension feedback. Front Robot AI 2017;4:17.

29. Edelmann J, Petruska AJ, Nelson BJ. Magnetic control of continuum devices. Int J Rob Res 2017;36:68-85.

30. Krings T, Finney J, Niggemann P, et al. Magnetic versus manual guidewire manipulation in neuroradiology: in vitro results. Neuroradiology 2006;48:394-401.

31. Lalande V, Gosselin FP, Vonthron M, et al. In vivo demonstration of magnetic guidewire steerability in a MRI system with additional gradient coils. Med Phys 2015;42:969-976.

32. Clogenson HC, Dankelman J, van den Dobbelsteen JJ. Steerable guidewire for magnetic resonance guided endovascular interventions. J Med Device 2014;8:021002.

33. Cha H-J, Yi B-J, Won JY. An assembly-type master-slave catheter and guidewire driving system for vascular intervention. Proc Inst Mech Eng H 2017;231:69-79. 
34. Kung Y-C, Huang K-W, Fan Y-J, et al. Fabrication of 3d high aspect ratio pdms microfluidic networks with a hybrid stamp. Lab Chip 2015;15:1861-1868.

35. Liu L, Zhang J, Badshah MA, et al. A programmable nanoreplica molding for the fabrication of nanophotonic devices. Sci Rep 2016;6:22445.

36. Zhang $\mathrm{M}, \mathrm{Wu} \mathrm{J}$, Wang $\mathrm{L}$, et al. A simple method for fabricating multi-layer pdms structures for $3 \mathrm{~d}$ microfluidic chips. Lab Chip 2010;10:1199-1203.

37. Jiao J, Sun L, Guo Z, et al. Antibacterial and anticancer PDMS surface for mammalian cell growth using the chinese herb extract paeonol (4-methoxy-2-hydroxyacetophenone). Sci Rep 2016;6:38973.

38. Schuerle S, Erni S, Flink M, et al. Three-dimensional magnetic manipulation of micro-and nanostructures for applications in life sciences. IEEE Trans Magn 2013;49: 321-330.

39. Kratochvil BE, Kummer MP, Erni S, et al. Minimag: A hemispherical electromagnetic system for 5-dof wireless micromanipulation. In: Experimental Robotics; 2014, pp. 317-329.

40. Kummer MP, Abbott JJ, Kratochvil BE, et al. OctoMag: an electromagnetic system for 5-dof wireless micromanipulation. IEEE Trans Rob 2010;26:1006-1017.
41. Dikkers R, Greuter M, Kristanto W, et al. Assessment of image quality of 64-row dual source versus single source ct coronary angiography on heart rate: a phantom study. Eur J Radiol 2009;70:61-68.

42. Lee YK, Jang KI, Ma Y, et al. Chemical sensing systems that utilize soft electronics on thin elastomeric substrates with open cellular designs. Adv Funct Mater 2017;27:1605476.

43. Mohammadi M, Poshtan M. Human motion energy harvesting by design of handheld linear pm synchronous generator. In: 3rd International Conference on Electric Power and Energy Conversion Systems (EPECS); 2013, pp. 1-6.

Address correspondence to: Hongsoo Choi Department of Robotics Engineering Daegu Gyeongbuk Institute of Science and Technology (DGIST) Daegu 42988 South Korea

E-mail: mems@dgist.ac.kr 